\title{
Evolutionary insights into heart regeneration
}

\author{
Jing-Wei Xiong
}

\begin{abstract}
Some lower vertebrates such as zebrafish and axolotl have incredible cardiac regenerative potential while mammals have very limited ones. Comparative studies among species have revealed that cardiomyocyte polyploidy, endothermy, and injury-induced activation of certain transcriptional factors including AP1 complexes are critical for cardiomyocyte proliferation and heart regeneration during animal evolution. Gaining insights into these evolutionarily conserved mechanisms will likely lead to achieving heart regeneration in non-regenerative mammals including humans.
\end{abstract}

Regenerative potential in the animal kingdom is a fundamental topic of regenerative biology and medicine. With the development of genome science and genetics technology, many investigators started to address how regenerative potential is lost or gained during the evolutionary courses of particular animal species. It is now documented that either wholeanimal or organ regeneration is achieved by activating adult stem cells (such as hydra and planarians; blood, skins, skeletal muscles, and livers in vertebrates), by inducing Muller glia reprogramming into neurons in adult zebrafish retinas, or by promoting cardiomyocyte (CM) proliferation in adult zebrafish hearts, and neonatal mouse, rat, and pig hearts (Beisaw et al. 2020; Duncan and Sanchez Alvarado 2019; Goldman and Poss 2020; Hoang et al. 2020; Tzahor and Poss 2017; Wang et al. 2020). In this short opinion article, we review several recent studies on evolutionary insights into heart regeneration that may be exploited for promoting non-regenerative heart regeneration.

Most mammalian cardiomyocytes (CMs) are mononuclear diploid CMs during development, but they become mostly polyploid CMs at late gestation or early postnatal stages. The appearance of CM polyploidy conincides with the loss of cardiac regenerative potential in mice, rats, and pigs (Gan et al. 2020). However, it remains largely unknown whether $C M$

Correspondence: jingwei_xiong@pku.edu.cn

Beijing Key Laboratory of Cardiometabolic Molecular Medicine, Institute of Molecular Medicine, and State Key Laboratory of Natural and Biomimetic Drugs, Peking University, Beijing 100871, China polyploidy is a causative factor for the loss of cardiac regenerative potential. A recent work has reported that the frequency of adult mononuclear diploid cardiomyocytes (MDCMs) is highly correlated to the cardiac regenerative potential in a large collection of 120 inbred mouse strains (Patterson et al. 2017). The authors meticulously assessed the percentage of MDCMs in 120 mouse strains, ranging from $2 \%$ to $18 \% \mathrm{MDCMs}$, and they then found that the more MDCMs, the better their hearts regenerate, with increased $\mathrm{CM}$ proliferation after myocardial infarction (MI). Genomewide association analysis has revealed that Tnni3k is associated with this phenotype, which is further supported by assessing the percentage of MDCMs, CM proliferation, and the distribution of diploid and polyploid CMs in cardiacspecific Tnni3k knockout mice after MI. Nevertheless, they did not find improvement in cardiac function and fibrosis in these mutant mice compared with control siblings post MI. On the other hand, they found that CMs are primarily diploids in zebrafish and cardiac-specific overexpression of zebrafish tnni3k leads to more CM polyploidy and loss of their regenerative capacity after ventricular resection. Similarly, transient inhibition of cytokinesis by cardiac-specific overexpression of dominant-negative Ect2 results in more CM polyploidy and compromised cardiac regeneration in zebrafish (Gonzalez-Rosa et al. 2018). Together, these works present new mechanistic insights into the causative effect of CM polyploidy on heart regeneration.
Springer Open Commons licence, unless indicated otherwise in a credit line to the material. If material is not included in the article's Creative Commons licence and your intended use is not permitted by statutory regulation or exceeds the permitted use, you will need to obtain permission directly from the copyright holder. To view a copy of this licence, visit http://creativecommons.org/ licenses/by/4.0/. The Creative Commons Public Domain Dedication waiver (http://creativecommons.org/publicdomain/zero/1. 0/) applies to the data made available in this article, unless otherwise stated in a credit line to the data. 
Another elegant work has reported that the loss of cardiac regenerative potential is highly related with $\mathrm{CM}$ polyploidization via phylogenetic analysis of $\mathrm{CM}$ nucleation and polyploidy in a large collection of non-vertebrates and vertebrates, and the percentage of MDCMs inversely correlates with metabolic rate, body temperature, and serum total thyroid hormone T4 levels (Hirose et al. 2019). They have further demonstrated that cardiac-specific inhibition of thyroid hormone receptor a (Thra) increases CM proliferation and the percentage of MNCMs in neonatal and adult mice, and results in an evident improvement in cardiac function and fibrosis after myocardial ischemic reperfusion in adult mice. Consistently, exogenous application of thyroid hormone T3 inhibits heart regeneration with evident $\mathrm{CM}$ polyploidization and decreased CM proliferation in zebrafish. Thus, thyroid hormone signaling promotes $\mathrm{CM}$ polyploidy and decreases cardiac regenerative potential during animal evolution.

As illustrated above, cardiac regenerative potential inversely correlates with $\mathrm{CM}$ polyploidization and postnatal endothermy/body temperature during animal evolution. While having learned a great deal of signaling pathways in regulating CM proliferation and heart regeneration, we still have very limited knowledge on the roles of non-coding DNA elements in heart regeneration, which are quite diverse during evolutionary species. Comparing with previous studies on searching for regenerative enhancers from different organ regeneration processes in zebrafish (Goldman et al. 2017; Kang et al. 2016; Thompson et al. 2020), Sánchez Alvarado and colleagues have recently reported the isolation of 49 conserved regenerative enhancers in the hearts and fins between killifish and zebrafish, which a good fraction of these 49 enhancers are also conserved in regenerative mouse strain acomys cahirinus but not non-regenerative mouse strain mus musculus (Wang et al. 2020). They have reported that the enhancer inhba is highly conserved between regenerative zebrafish (Z-IEN) and killifish (K-IEN) but not in non-regenerative humans (H-IEN), and this enhancer is activated in the progenitors of blastema during fin regeneration. Genetic depletion of this enhancer in killifish (K-IEN) suggests its essential roles in both heart and fin regeneration. Further bioinformatics analysis identified that AP-1 binding sites are enriched in these 49 regenerative enhancers and are also essential for regeneration. Together with other works (Beisaw et al. 2020; Gehrke et al. 2019), the AP-1 complexes appear to be evolutionarily conserved and injury-induced transcription factors for heart regeneration.

In brief, these recent studies have utilized comparative studies on heart regeneration among evolutionary species, leading to identifying critical factors such as thyroid hormones and cytokinesis factor (Tnni3k) for regulating $\mathrm{CM}$ nucleation and polyploidization, as well as noncoding DNA elements (regenerative enhancers) and binding factors (AP-1 complexes) for regulating $\mathrm{CM}$ proliferation. These and future studies will likely reveal a network of transcription factors and enhancers for coordinating $\mathrm{CM}$ proliferation and heart regeneration. In addition to these critical factors and regenerative enhancers, the field will take advantage of genome editing and chemical biology approaches to identify factors and small molecules that are sufficient for promoting nonregenerative heart regeneration in the coming years.

\section{Acknowledgements}

The author acknowledge Drs. Xiaojun Zhu and Qinchao Zhou for their comments on this manuscript, and funding supports from National Natural Science Foundation of China (31730061, 31430059).

Author's contributions

The author(s) read and approved the final manuscript.

Competing interests

The author declares that he has no competing interests.

Published online: 01 December 2020

\section{References}

Beisaw A, Kuenne C, Guenther S, Dallmann J, Wu CC, Bentsen M, Looso M, Stainier DYR. AP-1 contributes to chromatin accessibility to promote sarcomere disassembly and Cardiomyocyte protrusion during Zebrafish heart regeneration. Circ Res. 2020;126:1760-78.

Duncan EM, Sanchez Alvarado A. Regulation of genomic output and (Pluri) potency in regeneration. Annu Rev Genet. 2019;53:327-46.

Gan P, Patterson M, Sucov HM. Cardiomyocyte polyploidy and implications for heart regeneration. Annu Rev Physiol. 2020;82:45-61.

Gehrke AR, Neverett E, Luo YJ, Brandt A, Ricci L, Hulett RE, Gompers A, Ruby JG, Rokhsar DS, Reddien PW, et al. Acoel genome reveals the regulatory landscape of whole-body regeneration. Science. 2019;363:eaau6173.

Goldman JA, Kuzu G, Lee N, Karasik J, Gemberling M, Foglia MJ, Karra R, Dickson AL, Sun F, Tolstorukov MY, et al. Resolving heart regeneration by replacement histone profiling. Dev Cell. 2017;40:392-404 e395.

Goldman JA, Poss KD. Gene regulatory programmes of tissue regeneration. Nat Rev Genet. 2020;21:511-25.

Gonzalez-Rosa JM, Sharpe M, Field D, Soonpaa MH, Field LJ, Burns CE, Burns CG. Myocardial Polyploidization creates a barrier to heart regeneration in Zebrafish. Dev Cell. 2018:44:433-46 e437.

Hirose K, Payumo AY, Cutie S, Hoang A, Zhang H, Guyot R, Lunn D, Bigley RB, Yu $\mathrm{H}$, Wang J, et al. Evidence for hormonal control of heart regenerative capacity during endothermy acquisition. Science. 2019;364:184-8.

Hoang, T., Wang, J., Boyd, P., Wang, F., Santiago, C., Jiang, L., Yoo, S., Lahne, M., Todd, L.J., Jia, M, et al. (2020). Gene regulatory networks controlling vertebrate retinal. regeneration. https://doi.org/10.1126/science.abb8598. Online ahead of print.

Kang J, Hu J, Karra R, Dickson AL, Tornini VA, Nachtrab G, Gemberling M, Goldman JA, Black BL, Poss KD. Modulation of tissue repair by regeneration enhancer elements. Nature. 2016;532:201-6.

Patterson M, Barske L, Van Handel B, Rau CD, Gan P, Sharma A, Parikh S, Denholtz M, Huang Y, Yamaguchi Y, et al. Frequency of mononuclear diploid cardiomyocytes underlies natural variation in heart regeneration. Nat Genet. 2017;49:1346-53.

Thompson JD, Ou J, Lee N, Shin K, Cigliola V, Song L, Crawford GE, Kang J, Poss $K D$. Identification and requirements of enhancers that direct gene expression during zebrafish fin regeneration. Development. 2020;147:dev191262.

Tzahor E, Poss KD. Cardiac regeneration strategies: staying young at heart. Science. 2017:356:1035-9.

Wang W, Hu CK, Zeng A, Alegre D, Hu D, Gotting K, Ortega Granillo A, Wang Y, Robb S, Schnittker R, et al. Changes in regeneration-responsive enhancers shape regenerative capacities in vertebrates. Science. 2020;369:eaaz3090. 